\title{
Comparison of Binary Models for the Associated Factors Affecting Recovery Status of Vesico-vaginal Obstetrics Fistula Patients: A Case of Mettu Hamlin Fistula Center, South West Ethiopian
}

\section{Aboma Temesgen*}

Department of Statistics, Haramaya University, Dire Dawa, Ethiopia

\begin{abstract}
Background: Obstetric fistula or vaginal fistula is a medical condition in which a fistula (hole) develops between either the rectum or vagina or between the bladder and vagina after severe or failed childbirth, when adequate medical care is not available. It is the most tragic of preventable childbirth complications in the developing world, as affected women are often abandoned by their husbands and family, and forced to live in shame.
\end{abstract}

Objective: The main objective the study was to determine an appropriate binary model for the recovery status of the vesico-vaginal patients. Furthermore, the study explores factors affecting the recovery status of the patients during the time period of the study.

Methods: The study consists of 206 vesico-vaginal fistula patients having all required information who were taking treatment at Mettu Hamlin Fistula center from November 2010 to June 2014. The chi-square test of association was employed to explore the association between the recovery status and categorical independent variables. After exploring the association between the variables, different binary models were employed to have an appropriate model for the recovery status of the patients based on Akaki information criteria of the model.

Results: The chi-square test of association showed that width of fistula length of fistula and bladder size categories were significantly associated with recovery status of the patients at $5 \%$ of level of significance. The study showed among the candidate binary models logistic model was considers an appropriate model. Furthermore, the fitted model showed width, length of fistula and bladder size categories were the factors that have significant effect on the recovery status of the patients at $5 \%$ level of significance.

Conclusion: Logistic regression model was the better fit of the data whereas the fistula patients with width and length fistula category group between three up to five centimeter were less likely to be recovered comparison with the fistula patients group with width and length of fistula less than or equal to two centimeters. Similarly, the none bladder size category patients where less likely recover in comparison with fair bladder size fair bladder size group patients.

Keywords: Binary data modeling; Akaki information criteria; Link function; Vesico-vaginal fistula

\section{Introduction}

Obstetric fistula or vaginal fistula is a medical condition in which a fistula (hole) develops between either the rectum or vagina or between the bladder and vagina after severe or failed childbirth, when adequate medical care is not available. It is considered a disease of poverty because of its tendency to occur in women in poor countries who do not have health resources comparable to developed nations [1].

It is classically regarded as an accident of childbirth in which prolonged obstructed labor leads to destruction of the vesico-vaginal/ rectovaginal septum with consequent loss of urinary and/or fecal control. Obstetric fistula is highly stigmatizing and afflicted women often become social outcasts. Although obstetric fistula has been eliminated from advanced industrialized nations, it remains a major public health problem in the worlds poorest countries. Several million cases of obstetric fistula are currently thought to exist in sub-Saharan Africa and south Asia [2].

Immediate causes for vesico-vaginal fistula may be obstructed labor, pelvic surgery, and sexual abuse before reaching physical maturity, malignancy, radiotherapy or a combination of these. In most third world countries over $90 \%$ of fistulas are of obstetric nature and usually caused by obstructed labor [3]. Whereas Primary risk factors include early or closely spaced pregnancies and lack of access to emergency obstetric care Women affected with Crohn's disease also have a higher risk of developing obstetric fistulas. It mainly occurs amongst the illiterate farmers after prolonged obstructed labor. Public enlightenment and appropriate ante-natal care and delivery would reduce the incidence [4].

Obstetric fistula is the most tragic of preventable childbirth complications in the developing world, as affected women are often abandoned by their husbands and family, and forced to live in shame. They occur almost entirely in the developing world and their incidence is poorly studied. As obstetric fistula is a serious preventable public health issue in developing countries, national and international organizations should launch a campaign to end fistula by increasing the resources and skilled staff available locally to treat obstetric fistula for improving the lives of women currently living with this condition.

*Corresponding author: Aboma Temesgen, Department of Statistics Haramaya University, Dire Dawa, Ethiopia, Tel: +125912859758; E-mail: aboma.temesgen@gmail.com

Received October 06, 2015; Accepted December 11, 2015; Published December 22, 2015

Citation: Temesgen A (2016) Comparison of Binary Models for the Associated Factors Affecting Recovery Status of Vesico-vaginal Obstetrics Fistula Patients: A Case of Mettu Hamlin Fistula Center, South West Ethiopian. J Biom Biostat 7: 273. doi:10.4172/2155-6180.1000273

Copyright: (c) 2016 Temesgen A. This is an open-access article distributed under the terms of the Creative Commons Attribution License, which permits unrestricted use, distribution, and reproduction in any medium, provided the original author and source are credited. 
Citation: Temesgen A (2016) Comparison of Binary Models for the Associated Factors Affecting Recovery Status of Vesico-vaginal Obstetrics Fistula Patients: A Case of Mettu Hamlin Fistula Center, South West Ethiopian. J Biom Biostat 7: 273. doi:10.4172/2155-6180.1000273

Page 2 of 7

Moreover, effective preventive strategies for obstetric fistula such as better education to women and provision of improved obstetric care and searching for the best approaches to both prevention and treatment should be the priority [5].

Besides physical treatment, mental health services are also needed to rehabilitate fistula patients, who experience a great deal of psychological trauma from being ostracized by her community and from fear of developing fistula again. A study on the first formal counseling program for fistula survivors in Eritrea shows positive results, whereby counseling significantly improved the women's selfesteem, knowledge about fistula and fistula prevention, and behavioral intentions for health maintenance and social reintegration following surgery [6]. Cross-sectional study in Ethiopia also showed fistula prevalence of the disease among 19, 153 households with 97, 765 inhabitants surveyed fifty-five women with fistula were identified, of which 39 untreated. The overall estimated prevalence of fistula was 2.2 per 1000 women [7].

\section{Binary data modeling}

The binary model is among generalized linear models in which the outcome variables are measured on a binary scale that is the responses may be form of recovered or not recovered, alive or dead and present or absent. This binary data modeling is widely used in medical literature especially for correlating the dichotomous outcomes with the predictor variables that include different physiological data. In case of binary logistic regression, the predicted odd ratio of positive outcome is expressed as a sum of product. Product is formed by multiplying the values of independent variable and its coefficients. The probability of positive outcome is obtained from the odd ratio through a simple transformation $[8,9]$.

A logistic regression model is the result of non-linear transformation of the linear regression model. The difference between logistic regression and linear regression is that the outcome variable in logistic regression is dichotomous [10]. Many medical research problems call for the analysis and prediction of a dichotomous outcome: whether smokers will have a chance of developing lung cancer, hyperuricemia patients have the risk of getting cardio vascular disease. Traditionally, these research questions were addressed by either ordinary least squares (OLS) regression or linear discriminant function analysis [11].

The recovery status of the patient from the vesico-vaginal obstetrics fistula was also one of the binary outcome variables that is the patients recovered or not recovered from the disease after getting treatment during the study period. However, the previous studies conducted on vesico-vaginal fistula were on the measures of prevalence the diseases in Ethiopian context rather than modeling to determine factors related to the prevalence of fistula. Therefore, the main aim of this study was to investigate an appropriate binary model for the recovery status of the vesico-vaginal patients in the study area. Furthermore, the study also explores factors that determine the recovery status of the patients.

\section{Methodology}

\section{Data sources}

Data for this study was obtained from the retrospective institutional based study conducted on mothers came for fistula repair at Mettu Hamlin fistula center from November 2010 to June 2014 G.C. The study considered all women who visited at the center having vesico-vaginal fistula case only during the time period as study population. Among the total of 585 women patients visited from the year November 2010 to June 2014 G.C 206 having complete information was considered in the study.

Variables of the study: The outcome variable of the study was the recover status from vesico-vaginal fistula patients which indicates wether the patient was recovered from the vesico-vaginal fistula or not recovered during the time period. A patient is said to be recovered if she physically cured from her sickness and no requirement for intervention of health care professionals otherwise we call it the patient is not recovered during the study period.

The study also considered 19 independent variables to know their significant effects on the recover status of the patients. The list of this independent variables with respective of their categories was given on the Table 1 below.

\section{Binary data analysis}

It is common to have binary outcome variables in many medical cases that is a patient get cured or not, recovered or not and died or not from their disease after getting certain treatments. This study also deals with recovery status of the vesico-vaginal patient after getting treatment to know whether the patient is recovered or not during the study period. That is the outcome variable considered for the study was binary in nature as stated under the variables of the study section of the methodology part. Therefore, the outcome variable takes the value of 1 if the patients recovered from vesico-vaginal fistula after getting treatment during the time period and 0 if the patient is note recovered from the vesico-vaginal fistula obstetrics after having treatments during specified time period.

As the principal objective of this study was to investigate an appropriate binary model data in order to determine the relationship between the outcome variable with different predictor variables. This helps us to know the effect of this predictor on probability of recovering from vesico-vaginal fistula. That is the out come variable have binomial distribution with recovery from fistula obstetrics as success and not recovering from specified disease as failure event which can be expresses as follows:

\begin{tabular}{|c|c|}
\hline Variable & Categories of variables with their codes \\
\hline Age at first marriage & $0=\geq 15,1=16-19,2=\geq 20$ years \\
\hline $\begin{array}{l}\text { Age at occurrence of } \\
\text { VVF }\end{array}$ & $0=\leq 20,1=21-29,2=\geq 30$ years \\
\hline $\begin{array}{l}\text { Weight of patient at } \\
\text { arrival in MHFC }\end{array}$ & $0=<50,1=\geq 50 \mathrm{~kg}$ \\
\hline Parity & $\begin{array}{l}\text { 0=Primipara, } 1=\text { Multipara, } 2=\text { Grandmultipara, } 3=\text { Nuli } \\
\text { para }\end{array}$ \\
\hline Educational status & $0=$ Illiterate, $1=$ Literate \\
\hline Marital status & $\begin{array}{l}0=\text { Single }, 1=\text { Married, } 2=\text { Divorced, } 3=\text { Widowed, } \\
4=\text { Separated }\end{array}$ \\
\hline Accompanying person & $\begin{array}{l}0=\text { self, } 1=\text { husband, } 2=\text { relatives, } 3=\text { husband and } \\
\text { relatives, } 4=\text { others }\end{array}$ \\
\hline Antenatal care & $0=$ Yes, $1=$ No \\
\hline Place of delivery & $0=$ Home, $1=$ Health institution, $2=$ Other \\
\hline Mode of delivery & $0=$ Vaginal, $1=$ Others \\
\hline Duration of incontinence & $0=\leq 3,1=4-6,2=\geq 7$ month \\
\hline Duration of labor & $0=\leq 2,1=3,2=\geq 4$ day \\
\hline Fetal outcome & $0=$ Still birth, $1=$ Alive, $2=$ Early neonatal, 3=Dead \\
\hline Width of fistula hole & $0=\leq 2,1=3-5,2=>5 \mathrm{~cm}$ \\
\hline Length of fistula hole & $0=\leq 2,1=3-5,2=>5 \mathrm{~cm}$ \\
\hline Status of urethra & $0=$ Intact, $1=$ Partially damaged, $2=$ Complete destructed \\
\hline Bladder size & $0=$ None, $1=$ Small, $2=$ Fair, $3=$ Good, $4=$ No information \\
\hline
\end{tabular}

Table 1: List of independent variables considered for the study. 
Citation: Temesgen A (2016) Comparison of Binary Models for the Associated Factors Affecting Recovery Status of Vesico-vaginal Obstetrics Fistula Patients: A Case of Mettu Hamlin Fistula Center, South West Ethiopian. J Biom Biostat 7: 273. doi:10.4172/2155-6180.1000273

Page 3 of 7

$Y_{i}= \begin{cases}1, & \text { for recovered patients } \\ 0, & \text { other wise }\end{cases}$

Where; Yi is the treatment outcome for the ith patient

Chi-square test of association: Before proceeding to the binary data modeling the study explored the association between the outcome variable and different categorical covariates using chi-square test of association. This test statistic was con- sidered for the study to verify whether the categorical covariates and the outcome variable are independent or not.

Hypothesis to be tested for the study was expressed as: Null hypothesis which states as the recovery status of the patients and the independent categorical variables are independent versus the alternative hypothesis which states the recovery status and categorical independent variables are dependent (not the null hypothesis) where the test statistics to test the hypothesis was expresses as:

$$
\chi^{2}=\sum_{j=1}^{r} \sum_{i=1}^{c} \frac{\left(0_{i j}-E_{i j}\right)^{2}}{E_{i j}} \sim \chi(\mathrm{r}-1)(\mathrm{c}-1)(\alpha)
$$

Where; Oij is observed value in jth row and ith column whereas

$$
\text { Eij }=\frac{\text { rowtotal } * \text { columntotal }}{\text { grandtotal }}
$$

which have chi-square distribution. Moreover, it can be verified as there is an association if the computed chi-square value is greater than the critical value of chi-square or if the computed probability of observing greater computed chi-square value (p-value) is less than the level of significance (alpha value). Meaning that the categorical outcome variable and the categorical independent variables were dependent

Modeling the recovering status from vesico-vaginal fistula: After identifying the associations between the predictors (independent variables) and the outcome variable using the chi-square test of independency the next step is to model the recovery status of the patients. This fitted model helps us to know the effects of the predictors which have an association on the probability of recovering from the diseases. Therefore, to have an appropriate model that represents recovery probability of the patients the study considered probit, complementary log-log and logistic regression models. Whereas the probability of recovering from the diseases was expressed as follows in case of logistic regression with probit link function:

$$
\begin{aligned}
& \pi_{i}=\frac{\exp \left(X^{T} \beta\right)}{1+\exp \left(X^{T} \beta\right)} \\
& \eta=\log \left(\frac{\pi i}{1-\pi_{i}}\right)=X^{T} \beta
\end{aligned}
$$

Where; $\eta$ is the logit link functions that associate the outcome variables with predictor variables as linear relation, $\mathrm{X}$ is nxp matrices of predictors and $\beta$ is px 1 vectors of coefficients for the predictor variables. Furthermore, there is also alternative way of modeling probability of recovering from the diseases in case of using probit and logit link functions where they can be expressed as:

$$
\begin{aligned}
& \pi_{i}=\int_{-\infty}^{X^{T} \beta} \frac{1}{\sqrt{2 \pi}} \exp \left(-\frac{1}{2} z^{2}\right) d z \\
& \eta=\phi^{-1}\left(\pi_{i}\right)=X^{T} \beta
\end{aligned}
$$

Where; here $\eta$ is the probit link functions that associate the outcome variables with predictor variables as linear relation using the inverse of cumulative standardized normal distribution whereas the complementary log log link function is expressed as follows:

$$
\begin{aligned}
& \pi_{i}=1-\exp \left(-\exp \left(X^{T} \beta\right)\right. \\
& \eta=\log \left(-\log \left(1-\pi_{i}\right)=X^{T} \beta\right.
\end{aligned}
$$

Model estimation techniques: The outcome variable which represents the treatment outcome of the patients are assumed to be the observed values of independent Bernoulli random variables $y_{1}, y_{2}, \ldots, y_{n}$ such that $y_{i}$ has the binomial distribution since it is observed from the nth Bernoulli process randomly. When these binary data are grouped by covariate class, the outcome variable have the form $\frac{y_{i}}{m_{i}}, \ldots \frac{y_{n}}{m n}$ where $0<\mathrm{y}_{\mathrm{i}}<\mathrm{m}_{\mathrm{i}}$, is the number of successes out of the $\mathrm{m}$, subjects in the ith covariate class. The vector of covariate class sizes $m=\left(m_{i}, \ldots, m_{n}\right)$ is called the binomial index vector or binomial denominator vector. Therefore, $\mathrm{Y}_{\mathrm{i}}$ indexed by $\mathrm{mi}$ with parameter $\pi_{\mathrm{i}}$ probability of recovering from VVF where its log likelihood functions is expressed as:

$$
L\left(y_{i}, \pi_{i}\right)=\prod_{i=1}^{n} P\left(y_{i}\right)=\prod_{i=1}^{n}\left(\begin{array}{l}
m_{i} \\
y_{i}
\end{array}\right) \pi^{y_{i}}\left(1-\pi_{i}\right)^{m_{i}-y_{i}}=\prod_{i=1}^{n}\left(\begin{array}{l}
m_{i} \\
i
\end{array}\right)\left(\frac{\pi_{i}}{1-\pi_{i}}\right)^{y_{i}}\left(1-\pi_{i}\right)^{m_{i}}
$$

Where the log likelihood function has the following forms:

$$
l\left(y_{i}, \pi_{i}\right)=\sum_{i=1}^{n}\left(\left(\begin{array}{c}
m_{i} \\
y_{i}
\end{array}\right)+y_{i} \log \left(\frac{\pi_{i}}{1-\pi_{i}}\right)+m_{i} \log \left(1-\pi_{i}\right)\right)
$$

The constant function of $\mathrm{y}_{\mathrm{i}}$ not involving $\pi_{\mathrm{i}}$, that is $\left(\begin{array}{c}m_{i} \\ y_{i}\end{array}\right)$ has no role in the estimation of $\pi_{i}$ we remove from the log likelihood function is reduced to:

$$
l\left(y_{i}, \pi_{i}\right)=\sum_{i=1}^{n}\left(y_{i} \log \left(\frac{\pi_{i}}{1-\pi_{i}}\right)+m_{i} \log \left(1-\pi_{i}\right)\right)
$$

But here we consider the three systematic parts (link function) of the model specifies the relation between the $\pi$ and the matrices $\mathrm{nxp}$ of covariates $\mathrm{X}$. consider the case of logil link function where;

$$
\begin{aligned}
\eta=\log \left(\frac{\pi_{i}}{1-\pi_{i}}\right) \text { in which } \pi_{i}=\frac{\exp \left(X^{T} \beta\right.}{1+\exp \left(X^{T} \beta\right.} \\
\quad l\left(\beta, y_{j}\right)=\sum_{i=1}^{n} \sum_{j=1}^{r} y_{i} X_{i j} \beta_{j}-\sum_{i=1}^{n} m_{i}\left(1+\exp \left(\sum_{j=1}^{r} X_{i j} \beta_{j}\right)\right)
\end{aligned}
$$

which obtained by substituting the value of probability of recovery from the logit link function where $\beta \mathrm{j}$ rxl vectors of the coefficient for the covariates which is obtained by maximizing the log likely hood function with respect to $\beta$ values. Similarly the loglikely hood function for the probit and complement lo-log link function is obtained by substituting the value of the probability of recovery from the given link function to estimate the parameters of the model.

Model adequacy tests: To have appropriate predictors as well as appropriate link function for the recovery probability from Visco Vaginal fistula various model selection criteria was considered which was discussed here under the following The Wald test: The Wald test was considered to test the significance of individual parameter in the estimated model to identify whether the covariate have significant effect on the recovery visco vaginal fistula or not. The fore the wald test statistics was expresses as:

$$
Z=\frac{\beta_{j}}{S E\left(\beta_{j}\right)} \sim N(0,1)
$$

Alternatively, we can treat the square of this statistic as approximately a chi-squared with one degree of freedom which has the following form that is $Z^{2} \sim \chi_{1}^{2}$ 
Depending on the wald test statistics if the covariates do not have significant on the recovery status of the patients that covariate was removed from the model depending on the values of computed Wald test statistics

Deviance: deviance value is another selection criteria considered for the model selection where it values is based on log likelihood and the model with minimum values of deviance statistics was considered as an appropriate model. The deviance value is used to compare nested models where the deviance value of a model expressed as:

$$
D=2 \varphi\left(l_{\text {full }}-l_{\text {current }}\right)
$$

Where, $\varphi$ represent the scale parameter lcurrent the log-likelihood of the current fitted model and lf ull is the log-likelihood of the saturated model.

The Akaike information criterion (AIC): Akaike information is also another model selection criteria considered for the selection of appropriate model as well as appropriate predictor covariates for probability of recover from VVF in the study area. Where the values of AIC is expressed as:

$$
A I C=-2 \log (\text { likelyhood })+2 p
$$

Where, $\log$ (likelihood) is the log likelihood function which measures the goodness of the fitted model where as $p$ is the number of estimated parameter in the model which measures the how complexity of fitted model.

We model with minimum values of AIC was considered as an appropriate model.

\section{Results}

\section{Descriptive result}

Among total of 206 vesico-vaginal fistula patients considered from November 2010 to June 2014 at Mettu Hamlin fistula Hospital most of the patients (157 (76.21\%)) of the patients were recovered whereas only $49(23.79 \%)$ were not recovered during the follow up period. The age at occurrence of fistula category with the recovery status of patients also shows most of $(63(40.1 \%))$ were the patients infected with the disease at an age of less than or equal to 15 years whereas most none recovered patients $(21(42.9 \%))$ also belongs to this age category group.

The age at marriage description also shows most of the patients (26 $(53.1 \%))$ of none recovered and most $(72(45.9 \%))$ recovered patients married at an age of less than or equal to 15 years. Similarily, $11(22.4 \%)$ of none recovered and 35 (22.3\%) of the recovered patients married at an age of greater than or equal to 20 years and between 16 and 19 years respectively which represents smaller proportion in comparison with other marriage age category groups.

Among the total patients considered in the study 30 (61.2\%) among the none recovered patients and 93 (59.2\%) among the recovered patients gets antenatal care which represents larger proportion of the patients in both groups in comparison with patients who had no antenatal care. Duration of lasting inconsistence description also indicates 29 (59.2\%) among the none recovered patients and 88 (56.1\%) recovered patients lasted duration of inconsistency between four up to six months in comparison with remaining periods of inconsistency groups. Similarily, $30(61.2 \%)$ among the none recovered fistula patients and $8(51.6 \%)$ among the recovered patients lasted duration of labor up to greater than four days which represents larger proportion in comparison with other categories of duration of labor.
Similarly the place of delivery of patients shows for both recovered and none recovered patients most $26(53.1 \%)$ of the none recovered patients and most $104(66.2 \%)$ among the recovered patients give the delivery at their home which represents the larger proportion of the patients in both cases in comparison with patient group who gave delivery at health institution.

It can be also observed from the Table 2 that among none recovered patients most $32(65.3 \%)$ of and among the recovered patients $138(87.9 \%)$ of them have length of fistula less than or equal to two centimeter which is larger proportion in comparison with remaining fistula length patients groups. Similarly as observed from the urethra status of the patients more than half of the urethra of none recovered patients were partially damaged whereas larger 97 (61.8\%) proportion of recovered patients urethra was intact in comparison with the remaining status of urethra groups.

\section{Exploring association between the recovery status and independent variables}

Before proceeding to the binary modeling first of all chi-square test of association were employed to explore whether the independent covariates have an association with treatment outcome or not Therefore, the chi-square test result of Table 3 showed that among all covariate considered in the study length of fistula, bladder size and width fistula categories have significant association with the recovery status of the patients at $5 \%$ level of significance since the probability of getting larger computed chi-square value for these covariates were smaller than 5\% level of significance. Similarly, weight categories and place of delivery were significance associated with recovery status at $10 \%$ level of significance since of the probability of observing larger computed value of chi-square for these covariates were lesser than $10 \%$ level of significance.

\section{Comparison of binary models}

After exploring the association between the recovery status and independent variables the three commonly used link function were considered for the binomial distribution model. Therefore; the entire three models were fitted with length of fistula, bladder size, weight of the patients and place of delivery and width of fistula as independent covariate since they have significant association with outcome variable the result of the model was also follows (Table 4).

As it can be observed from the log likelihood function and AIC of the three fitted models there is no this much bigger model criteria difference between the three fitted model results. But when we compare them the model with logit link function have smaller negative values of $\log$ likelihood and AIC value than probit and complementary log$\log$ link functions. Therefore, logit link function was considered as an appropriate link function to fit data in the study area than the probit and complementary log-log link functions.

\section{Factors affecting the recovery status of the patients}

The logistic model was fitted with an appropriate covariates and weight of the patients were removed from the model because of it do not have significant effects on the model. This model was fitted with width of fistula, length of fistula, bladder size and place of delivery as independent covariates. Among all the covariate considered in the model only place of delivery categories do not have significant effects on the recovery status at $5 \%$ level of significance (Table 5).

The estimated coefficient of logit model for the width of fistula category group three up to five centimeter -0.774 indicates that $\log$ 
Citation: Temesgen A (2016) Comparison of Binary Models for the Associated Factors Affecting Recovery Status of Vesico-vaginal Obstetrics Fistula Patients: A Case of Mettu Hamlin Fistula Center, South West Ethiopian. J Biom Biostat 7: 273. doi:10.4172/2155-6180.1000273

Page 5 of 7

\begin{tabular}{|c|c|c|c|c|}
\hline Covariates & Categories & Recovery status & & Total n (\%) \\
\hline & & Not recovered n (\%) & Recovered n (\%) & \\
\hline \multirow[t]{3}{*}{ Age at occurrence of fistula } & $<=15$ years & $21(42.9)$ & $63(40.1)$ & $84(40.78)$ \\
\hline & $16-19$ years & $18(36.7)$ & $56(35.7)$ & $74(35.92)$ \\
\hline & $>=20$ years & $10(20.4)$ & $38(24.2)$ & $48(23.30)$ \\
\hline \multirow[t]{3}{*}{ Age at marriage } & $<=15$ years & $26(53.1)$ & $72(45.9)$ & $98(47.57)$ \\
\hline & $16-19$ years & $12(24.5)$ & $35(22.3)$ & $47(22.82)$ \\
\hline & $>=20$ years & $11(22.4)$ & $50(31.8)$ & $61(29.61)$ \\
\hline \multirow[t]{2}{*}{ Height } & $<=150$ Centimeter & $44(89.8)$ & $129(82.2)$ & $173(83.98)$ \\
\hline & $>150$ Centimeter & $5(10.2)$ & $28(17.8)$ & $32(15.53)$ \\
\hline \multirow[t]{2}{*}{ Weight } & $<50$ kilogram & $43(87.8)$ & $117(74.5)$ & $160(77.67)$ \\
\hline & $>=50$ kilogram & $6(12.2)$ & $40(25.5)$ & $46(22.33)$ \\
\hline \multirow[t]{3}{*}{ parity } & Primipara & $4(8.2)$ & $21(13.4)$ & $25(12.14)$ \\
\hline & Multipara & $28(57.1)$ & $76(48.4)$ & $104(50.49)$ \\
\hline & Grandmultipara & $17(34.7)$ & $60(38.2)$ & $77(37.38)$ \\
\hline \multirow[t]{2}{*}{ Educational status } & Illiterate & $10(20.4)$ & $50(31.8)$ & $60(29.13)$ \\
\hline & Literate & $39(79.6)$ & $107(68.2)$ & $146(70.87)$ \\
\hline \multirow[t]{4}{*}{ Marital status } & Single & $4(8.2)$ & $6(3.8)$ & $10(4.85)$ \\
\hline & Married & $28(57.1)$ & $104(66.2)$ & $132(64.08)$ \\
\hline & Divorced & $11(22.4)$ & $39(24.8)$ & $50(24.27)$ \\
\hline & Separated & $6(12.2)$ & $8(5.1)$ & $14(6.80)$ \\
\hline \multirow[t]{5}{*}{ Accompanying person } & Self & $2(4.1)$ & $6(3.8)$ & $8(3.88)$ \\
\hline & Husband & $15(30.6)$ & 47 (29.9) & $62(30.10)$ \\
\hline & Relative & $10(20.4)$ & $39(24.8)$ & $49(23.79)$ \\
\hline & Husband and relatives & $4(8.2)$ & $6(3.8)$ & $10(4.85)$ \\
\hline & Others & $18(36.7)$ & $59(37.6)$ & $77(37.38)$ \\
\hline \multirow[t]{2}{*}{ Antenatal care } & Yes & $30(61.2)$ & $93(59.2)$ & $123(59.71)$ \\
\hline & No & $19(38.8)$ & $64(40.8)$ & $83(40.29)$ \\
\hline \multirow[t]{3}{*}{ Duration of incontinence } & $<=3$ month & $8(16.3)$ & $24(15.3)$ & $32(15.53)$ \\
\hline & 4-6 month & $29(59.2)$ & $88(56.1)$ & $117(56.80)$ \\
\hline & $>=7$ month & $12(24.5)$ & $45(28.7)$ & $57(27.67)$ \\
\hline \multirow[t]{3}{*}{ Duration of labor } & $<=2$ days & $14(28.6)$ & $52(33.1)$ & $66(32.04)$ \\
\hline & 3-4 days & $5(10.2)$ & $24(15.3)$ & $29(14.08)$ \\
\hline & $>=4$ days & $30(61.2)$ & $8(51.6)$ & $38(18.45)$ \\
\hline \multirow[t]{2}{*}{ Place of delivery } & Home & $26(53.1)$ & $104(66.2)$ & $130(63.11)$ \\
\hline & Health institutions & $23(46.9)$ & $53(33.8)$ & $76(36.89)$ \\
\hline \multirow[t]{2}{*}{ Mode of delivery } & aginal & $16(32.7)$ & $68(43.3)$ & $84(40.78)$ \\
\hline & Others & $33(67.3)$ & $89(56.7)$ & $122(59.22)$ \\
\hline \multirow[t]{2}{*}{ Fetal out come } & Still birth & $43(87.8)$ & $139(88.5)$ & $182(88.35)$ \\
\hline & Alive & $6(12.2)$ & $18(11.5)$ & $24(11.65)$ \\
\hline \multirow[t]{2}{*}{ Length of fistula hole } & $<=2$ centimeter & $32(65.3)$ & 138 (87.9) & $170(82.52)$ \\
\hline & 3- 5 centimeter & $17(34.7)$ & $19(12.1)$ & $36(17.48)$ \\
\hline \multirow[t]{2}{*}{ Width of fistula } & $<=2$ centimeter & $25(51.0)$ & $118(75.2)$ & $143(69.42)$ \\
\hline & 3-5 centimeter & $24(49.0)$ & $39(24.8)$ & $63(30.58)$ \\
\hline \multirow[t]{3}{*}{ Bladder size } & None & $25(51.0)$ & $101(64.3)$ & $126(61.17)$ \\
\hline & Small & $18(36.7)$ & $52(33.1)$ & $70(33.98)$ \\
\hline & Fair & $6(12.2)$ & $4(2.5)$ & $10(4.85)$ \\
\hline \multirow[t]{2}{*}{ Status of urethra } & Intact & $24(49.0)$ & $97(61.8)$ & $121(58.74)$ \\
\hline & Partially damaged & $25(51.0)$ & $60(38.2)$ & $85(41.26)$ \\
\hline Total & - & $49(23.79)$ & $157(76.21)$ & $206(100.00)$ \\
\hline
\end{tabular}

Table 2: Description of independent variables with recovery status of the patients. 
Citation: Temesgen A (2016) Comparison of Binary Models for the Associated Factors Affecting Recovery Status of Vesico-vaginal Obstetrics Fistula Patients: A Case of Mettu Hamlin Fistula Center, South West Ethiopian. J Biom Biostat 7: 273. doi:10.4172/2155-6180.1000273

Page 6 of 7

\begin{tabular}{|c|c|c|c|c|c|c|c|}
\hline Covariate & DF & Chi-square value & p-value & Covariate & DF & Chi-square value & $p$-value \\
\hline Current age & 2 & 0.311 & 0.856 & Age at marriage & 2 & 1.600 & 0.499 \\
\hline Height & 1 & 1.616 & 0.204 & Weight & 1 & 3.77 & $0.052^{*}$ \\
\hline Parity & 2 & 1.524 & 0.467 & Education & 1 & 2.367 & 0.124 \\
\hline Marital status & 3 & 4.829 & 0.185 & Accompany person & 4 & 1.778 & 0.777 \\
\hline Antenatal care & 1 & 0.061 & 0.804 & Duration of inconsistency & 2 & 0.326 & 0.804 \\
\hline Duration of labor & 2 & 1.57 & 0.456 & Place of delivery & 1 & 2.787 & $0.095^{*}$ \\
\hline Mode of delivery & 1 & 1.757 & 0.185 & Fetal outcome & 1 & 0.022 & 0.882 \\
\hline Length of fistula & 1 & 13.217 & $0.000^{* *}$ & Width of fistula & 1 & 10.250 & $0.001^{* *}$ \\
\hline Bladder size & 2 & 8.459 & $0.015^{\star *}$ & Status of urethra & 1 & 2.526 & 0.112 \\
\hline
\end{tabular}

NB:** Indicates significance at $5 \%$ level of significance, ${ }^{*}$ Indicates significance at $10 \%$ level of significance

Table 3: Chi-square test results for independence.

\begin{tabular}{|c|c|c|c|}
\hline Link function & DF & Log likelihood function & AIC \\
\hline Logit & 6 & -100.771 & 213.541 \\
\hline Probit & 6 & -101.029 \\
\hline Clog-log & 6 & -101.140 \\
\hline
\end{tabular}

Table 4: Comparison of the models.

\begin{tabular}{|c|c|c|c|c|}
\hline Covaraites & Estimated coeff (SE) & $95 \%$ confidence & Exp (coeff) & $95 \%$ confidence \\
\hline Intercept & $2.092(0.332)$ & {$[1.477,2.784]^{* *}$} & 8.100 & {$[4.379,16.187]^{\star *}$} \\
\hline \multicolumn{5}{|l|}{ Width of fistula } \\
\hline \multicolumn{5}{|l|}{$<=2$ centimeter } \\
\hline $3-5$ centimeter & {$[-0.774(0.379)$} & {$[-1.517,-0.025]^{\star *}$} & 0.461 & {$[0.219,0.975]^{\star \star}$} \\
\hline \multicolumn{5}{|l|}{ Length of fistula } \\
\hline \multicolumn{5}{|l|}{$<=2$ centimeter } \\
\hline $3-5$ centimeter & $-1.060(0.424)$ & {$[-1.892,-0.223]^{\star *}$} & 0.346 & {$[0.151,0.800]^{\star *}$} \\
\hline \multicolumn{5}{|l|}{ Bladder size } \\
\hline \multicolumn{5}{|l|}{ None } \\
\hline Small & $-0.245(0.373)$ & {$[-0.974,0.497]$} & 0.783 & {$[0.378,1.644]$} \\
\hline Fair & $-1.622(0.718)$ & {$[-3.107,-0.223]^{\star \star}$} & 0.197 & {$[0.045,0.800]^{\star *}$} \\
\hline \multicolumn{5}{|l|}{ Place of delivery } \\
\hline \multicolumn{5}{|l|}{ Health institution } \\
\hline Home & $-0.561(0.362)$ & {$[-1.274,0.152]$} & 0.571 & {$[0.280,1.164]$} \\
\hline
\end{tabular}

** Indicates significance at $5 \%$ level of significance

Table 5: Logistic model result with estimated odd ratio.

Logit estimates odds ratio estimates

of odd ratio of recovery was 0.774 lower in this category group in comparison with patient group with fistula width less than or equal to two centimeter. Similarly, the estimated logit coefficient of bladder size with small category group -0.245 shows the $\log$ f odd ratio of patient group with bladder size small was 0.245 lower whereas estimated coefficient -1.622 for fair bladder size shows the log of odd ratio of patient group in this category group have 1.622 lower in comparison with none bladder size patient category.

The estimated odd ratio for three up to five width fistula 0.461 shows the odds recovery in this category group was 0.461 times less likely than the patient group with width of fistula less than or equal to two centimeter. Similarly, the estimated odd ratio for fair bladder size patients 0.197 also show that the odds of recovery in this category group group was 0.197 time that of none bladder size patient groups that is the fair bladder size patient groups are less likely to recover than none bladder size patient groups.

\section{Conclusion}

Among all patients considered in the study during the time period the prevalence of recovery from visco vaginal fistula was $76.21 \%$.
Whereas width of fistula, length of fistula and bladder size categories were significantly associated with recovery status of the patients where weight and place of delivery of the patients associated with recovery status at $10 \%$ level of significance.

The logistic binary model was an appropriate in fitting the recovery status of the patients in comparison binary model with probit and complementary lo-log link function. Furthermore, the fitted model showed that width and length of fistula category group between three up to five centimeter and bladder size fair category group have significant effect on the recovery status of the patients.

The study also revealed that vesico-vaginal fistula patients with width and length category group between three up to five centimeter were less likely to be recovered in comparison with less than or equal to two centimeter width and length of fistula category group patients.

\section{References}

1. Browning A (2004) Obstetric fistula in Ilorin, Nigeria. PLOS Med 8: 369-377.

2. Wall LL (2012) Preventing obstetric fistulas in low-resource countries: insights from a Haddon matrix. Obstet Gynecol Surv 67: 111-121. 
Citation: Temesgen A (2016) Comparison of Binary Models for the Associated Factors Affecting Recovery Status of Vesico-vaginal Obstetrics Fistula Patients: A Case of Mettu Hamlin Fistula Center, South West Ethiopian. J Biom Biostat 7: 273. doi:10.4172/2155-6180.1000273

3. Hilton P (2001) Vesico-vaginal fistula new perspectives. Curr Opin Obstet Gynecol 13: 513-520

4. Mikah S, Daru P, Karshima J, Nyango D (2011) The burden of vesico-vaginal fistula in north central Nigeria. J West Afr Coll Surg 1: 50-62.

5. Dangal G, Thapa K, Yangzom K, Karki A (2013) Obstetric fistula in the developing World: An Agonising Tragedy. NJOG 8: 5-15.

6. Khaliah A, Janet M, Letu H, Elsa M, Jena D, et al. (2010) The role of counseling for obstetric fistula patients: lessons learned from Eritrea. Patient Education and Counseling 80: 262-265.

7. Muleta M, Fantahun M, Tafesse B, Hamlin E, Kennedy R (2007) Obstetric fistula in rural Ethiopia. East Afr Med J 84: 525-533.
8. Samanta B, Bird L, Kuijpers M, Zimmerman A, Jarvik G, et al. (2009) Prediction of proven- tricular leukoplakia: Selection of hemodynamic features using logistic regression and decision tree algorithms. Journal of Artificial Intelligence in Medicine 46: 217-231

9. Dobson AJ (2002) An introduction to generalized linear models. Canada.

10. Hosmer DW, Lemeshow S (2005) Applied Logistic Regression. WileyInterscience Publication, New York

11. Brijesh S (2011) Reporting dichotomous data using logistic regression in medical research. Nepal Journal of Epidemiology 1: 111-113. 